\title{
NON-HYDRODYNAMIC CONTRIBUTION TO THE CONCENTRATION DEPENDENCE OF THE SELF DIFFUSION OF INTERACTING BROWNIAN MACROPARTICLES -
}

\author{
George D.J. PHILLIES \\ Department of Chemistn The Untersin of Michgan 4m Arbur Michuan 48109. USA
}

Recened 6 August 1982

The generalized Langevin equation and the stationary requirement for an equilibrium ststem sre used to calculate she modification of the self (tracer) diffusion coefficient by direct interactions In the absence of hydrodinamic thi, modificatron of $D_{s}$ is cqual to the previously predicted modification of the mutual-diffusion cozfficient $D_{m}$ by the same effew though $D_{m}$ is also modified by direct interactions in another way The effect treated here mas ne vewed as being a change in the generalized Smoluchowski equation in which the free-particle diffusion coefficient $D_{0}$ is replaced by a dresied difiusion coefficient. and not as being a memory-function effect in the contentıonal generalized Smoluchoush equation

\section{Introduction}

In a suspension of interactıng brownian molecules. there are two operationally disunct. numerically unequal, diffusion coefficients. One of these. the mutual-diffusion coefficient. $D_{\mathrm{m}}$. describes the relasation of a concentration gradient. The other. the self-diffusion coefficient $D_{\text {. }}$ characterizes the motion of individual particles through a uniform solution. These coefficients hate been argued [e.g. ref [1]) to be related via the generalized Stokes-Einstein equation

$$
D_{\mathrm{m}}=D_{\mathrm{s}}(\partial \pi / \partial c)_{\mu_{\mathrm{u}}} T(1-\dot{\phi}) / K_{\mathrm{B}} T \text {. }
$$

where $(\partial \pi / \partial c)_{\mu_{0}} T$ is the osmotic compressibility. $Q$ is the solute volume fraction. and $K_{B}$ is Boltzmann's constant For solutions of macromolecules in which $D_{\mathrm{in}}, D_{3}$ and - may be obtaned from quasi-elastuc light scatterıng, tracer dıffusion and equilibrium centrifugation studies respectuvely. eq (1) agrees with experiment to better than $5 \%[0,3]$.

Recently, several theoretıcal studies have argued [4-6] that eq (1) ought not be correct. in that there are physical effects which modify the self-diffusion coefficient but not the mutual-diffusion coefficieni One writes

$$
D_{\mathrm{s}}=K_{\mathrm{B}} T / f_{\mathrm{s}}, \quad D_{\mathrm{m}}=\left(\partial \pi / \partial_{\mathrm{c}}\right)_{\mu_{\mathrm{o}} T}(1-\phi) / f_{\mathrm{m}} .
$$

For this discussion, it is convenient to divide all intermacromolecular forces into two types- the direct interactions (e.g. the hard-sphere potential) and the solvent-mediated. velocity-dependent, hydrodynamic interactions. In a model system with no hydrodynamic forces, it was predicted by Ackerson [4]. Dieterich and Peschel [5], and Marqusee [6] that the friction factor $f_{3}$ of the self-diffusion coefficient is modified by direct interactions However, these workers found that direct interactions do not modify the friction factor $f_{\mathrm{m}}$ of the mutual-diffusion coefficient unless hydrodynamic interactions are present. In contrast. with a different approach this author has argued that direct interactions do modify $f_{m}$ elen if hydrodynamic

+ This uork uas supported in part by the National Science Foundation under Grant CHE-7920389

0301-0104/83/0000-0000/\$03.00 1983 North-Holland 
interactions are absent [7]. If different effects contributed to $f_{\mathrm{V}}$ and to $f_{\mathrm{m}}$, one would expect that $f_{\text {, and }} f_{\mathrm{m}}$ could not in general be equal, as is implied by eq. (1)

This recent work on the friction factors $f$ should be contrasted with classical studies on the diffusion of brownian molecules [8], in which direct interactions were assumed to contribute the thermodynamic factor to $D_{m}$ and provide the statistical weighting factor for the (distance-dependent) hydrodynamic interactions. but were assumed not to make a contribution separate from the hydrodynamic one to the friction factor $f$. (A histonc exception is provided by the work of Mazo [9], who used the Kirkwood fluctuation-dissipation formula for $f$ to compute the effect of a screened electrostatic interaction on $f_{4}$. Perhaps because this work was applied to the diffusion of micelles rather than covalently bonded polymers, it has largely been overlooked. More recently, Deulın [10] and this author [11] have treated the non-hydrodynamic contribution of direct interactions to the viscosity and the Stokes" law drag coefficient.)

The purposes of this paper are: first, to show that the previously obtained non-hydrodynamic contribution to $f_{\mathrm{m}}$ also appears in $f$, and. second. to show that the results of refs $[4-7,11]$ are in fact conisistent with each other. Section 2 of this paper presents our general calculation, the results are discussed in section 3.

\section{Method and calculation}

Our approach is based on the generalized Langevin equation

$$
M \mathrm{~d} v_{t}(t) / \mathrm{d} t=-f v_{t}(t)-\nabla_{t} W^{\prime}+F_{t}(t),
$$

$v_{z}$ being the velocity of particle $t, f$ being its drag coefficient, $W^{v}$ being the total potential energy of the macroparticles, and $F_{t}(t)$ being the fluctuating force on macroparticle $t$ due to its interactions with the adjoining solvent molecules. $F$, has some very short correlation time $\tau_{\mathrm{H}}$, which corresponds to the shortest times over which the drag force is described by the $-f v_{t}(t)$ term. $\tau_{\mathrm{H}}$ should not be confused with the much longer time $\tau_{\mathrm{B}}=m / f$ which is the relaxation time for the particle velocity. The distinction between $\tau_{\mathrm{H}}$ and $\tau_{B}$ is treated in more detall by Rice and Gray [12]

Conventional experimental techniques, such as diaphragm diffusion and light-scattering spectroscopy, are limited to tımes $t \gg 10 \mathrm{~ns}$. Over these relatively long perıods, the inertia of a brownian particle is negligible, so that eq. (3) may be rewritten

$$
v_{1}(t)=v_{B_{1}}(t)+v_{1}(t) \text {. }
$$

where by definition $v_{I_{t}}(t)=-\nabla_{t} W^{2} / f$ and $v_{\mathrm{B} t}(t)=\overline{F_{t}(t)} / f$, the overstroke "-" " denoting the effect of the fluctuating force $\boldsymbol{F}$, over a period sufficiently long that the frequency dependence of the drag coefficient $f$ is unimportant. For an equilibrium system, stationarity appears to require that eq. (4) be equally valıd at all times, including $t=0$. It is sometimes [13] asserted that eq. (4) is not valid at $t=0$. since the brownian particles require some initial time to achieve their drift velocity $v_{1}$. It is here preferred to assume that the system was created at some tıme $t \ll 0$, so the partıcles' drift velocities at $t=0$ were attained durıng the moments before $t=0$. This assumption requires that the interparticle forces change appreciably only over times much longer than $\tau_{B}$, a condition which almost always obtains

The use of the form $-\nabla_{t} W^{N}$ to represent the intermacroparticle force implies that the interparticle potentıal is differentıable everywhere. This requirement is satisfied by any physical system. but not by a model hard-sphere suspension. However, if one views a hard sphere as a limiting case of a differentiable potential, and if $f$ is large enough that the conditıons of the previous paragraph are still satisfied, the results of this paper would still be relevant.

The work presented here rests on a careful analysis of the correlations between the brownian and 
Interactive velocities $v_{B}$ and $v_{1}$ at the same and different times. One often encounters the assertion that these velocities are always uncorrelated, hecause the interparticle force depends on the relatue positions of the particles. while in the absence of hydrodynamic interactions the fluctuating solvent-solute force $F_{s}(t)$ Is independent of the particle positions. so that

$$
\left\langle v_{\mathrm{B}_{t}}(r) v_{\mathrm{L}_{1}}(\tau)\right\rangle=0
$$

One does clearly have

$$
\left\langle v_{1}(t)\right\rangle=\left\langle v_{\mathrm{B},}(t)\right\rangle=\left\langle v_{1,}(t)\right\rangle=0
$$

(the brackets " \langle\rangle " denoting an ensemble average) because the fluid as a whole has rotatuonal isotropi For the calculations below. One is interested in averages taken "ith a given initial configuration of the macroparticles, since $v_{l}$ is only non-vanishing if one specifies the macroparticle configuration. An aver.dge over the positions and momenta of the solvent molecules for a fixed macroparticle configuration in a sub-ensemble average (heremafter denoted "- ") over a part of the system"s canonical ensemble

From time reversal invariance,

$$
\overline{v_{s}(t)}=0 . \quad \overline{v_{1}(t)}=-\nabla_{,} W^{2} .
$$

On combining eqs. (7) and (4), there follows the surprising result

$$
\overline{v_{\mathrm{Br}}(t)}=\overline{-v_{\mathrm{l}}(t)}=\nabla_{\mathrm{t}} W^{\prime} / f \text {. }
$$

Eq (8) at first sight seems inconsistent with the staustical independence of the particle posituons and the random forec $F_{,}(t)$. A proper interpretation of a result equivalent to eq (8) was first presented br Batehelor [14]. As the explanation is somewhat involved. we first show in general how a non-uniform particle density leads at later times to correlations between particle positions and particle brownian ielocities. The discussion will then be specialized to the case of a solution of interacting brou nian particles Consider the set of particles which at time $t$ lie in some surface $S$ Since their velocity-ielocity correlution ume has the non-zero value $\tau_{B}$. at a time $t-\epsilon, \epsilon \ll \tau_{B}$, the particles must have been located away from $S$ bs distances of order $-\epsilon \boldsymbol{v}$. Thus, particles in $\mathrm{S}$ at time $t$, which are moving to the left. must at $t-\epsilon$ hate been found to the right of $S$, and vice versa. If at $t-\epsilon$ each parucle had an equal likelihood of haing a leftwards or rightwards velocity. and if at $t-\epsilon$ the densities of particles on the left and night sides of $S$ were equai. then at time $t$ one would expect an isotropic distribution of velocitres for the particles ling within $S$ Contrarywise, if at time $t-\epsilon$ each partıcle had an equal likelihood of having a leftwards or rightwards velocity. but if at $t-\epsilon$ there were more particles on one side of $S$ than on the other. then at une $I$ one would find a net flux of particles within S: particles would be found moung from the region of higher concentration to the region of lower concentration more often than in the other direction. A non-umform position distribution at $t-\epsilon$, even when coupled to an isotropic velocity distribution at $t-\epsilon$. results in a non-1sotropic velocity distribution at a later time $t$

In a suspension of interacting brownian particles. the $N$-particle spatial-distribution function is not a constant; rather, the likelihood of finding a pair of partıcles some distance apart is determined by the Boltzmann factor $\exp \left(-\beta W^{2}\right)$. If the particle densits around some point is non-uniform. then at later times $\tau, t<\tau<\tau_{\mathrm{B}}$, the brownian velocities $\boldsymbol{v}_{\mathrm{B}}$ will become correlated $w_{1}$ th the initial non-umformittes in the particle density. The points where the particle density gradients are non-zero, and hence where the parucle positions and brownian velocities are correlated. are precisely the points where $w^{\prime}$ is non-i anishing. From the second equation of (7), these are also precisely the points where the interactive velocity $t_{1}$ is non-zero. A quantitative relation between the interactive velocity and the sub-ensemble a erage brow nian veiocity then follows either from a detailed calculation or from stationarity. If the Boltzmann statistical wetght is to be independent of time. the rate at which the interactive velocity creates concentration gradients must exactly 
cancel the rate at which the sub-ensemble average brownian velocity relaxes those same concentration gradients, as will occur if eq. (8) is correct.

In applying this argument to a solution of interacting particles, one must require that the surface $S$ moves with the local drift velocity $v_{1}$. In the argument of the preceding paragraph. one might be tempted to say that one has a "non-equilibrium" distribution for the brownian veloctly because one first had a "non-equilibrium" density distribution for the particles. However, this paper is concerned only with equiltbrium systems. Properly speaking, eq. (8) gives an equilhbrium correlation between macroparticle positions and the brownian component of the macroparticle velocity

Several other properties of the brownian velocity are worth noting. As has been emphasized by Pusey [15] in a dilute system $v_{i}(t)$ [and therefore, from our arguments, also $\overline{v_{\mathrm{B}}(t)}$ ] is much less than $v_{\mathrm{B}}(t)$, so if one writes

$$
v_{\mathrm{B}}(t)=\overline{v_{\mathrm{B}}(t)}+\delta v_{\mathrm{B}}(t)
$$

in general $v_{\mathrm{B}}(t) \approx \delta v_{\mathrm{B}}(t)$. For non-interactıng particles in the absence of an external potential, $\overline{v_{\mathrm{B}}(t)}=0$ and $v_{\mathrm{B}}(t)=\delta v_{\mathrm{B}}(t)$. Furthermore, from eq. (4) $v_{\mathrm{B}}$ is defined to be the difference between the total velocity of the particle (which is odd under time reversal) and the interactive velocity of the particle (which is defined in terms of the particle positions, and hence is even under time reversal). so $v_{\mathrm{B}}(t)$ is a variable without definite time reversal parity.

Having exhıbited the correlation between the brownian and interactive velocities. the self-diffusion coefficient of a suspension of interacting brownian particles is now calculated. The self (tracer) diffusion coefficient $D_{\mathrm{s}}$ describes the motion of labelled solute molecules through a background of unlabelled but otherwise identical solute molecules, the experiment being so arranged that the total concentration of solute molecules is everywhere the same. $D_{\mathrm{s}}$ may be related to the ensemble average displacement $r$ of a typical labelled solute molecule during the time interval $\Delta t$

$$
6 D_{\mathrm{s}} \Delta t=\left\langle|r|^{2}\right\rangle \text {. }
$$

If $\Delta t$ is too small, or so large that the increase in $|r|^{2}$ is obstructed by the walls of the container, $D_{\varsigma}$ is no longer a simple constant. Since $r$ is the integral of the particle velocity $v(r)$, one has

$$
6 D_{9} t=\left\langle\int_{0}^{t} \mathrm{~d} s_{1} \int_{0}^{t} \mathrm{~d} s_{2} v\left(s_{2}\right)\right\rangle \text {. }
$$

The velocity correlation function in (11) depends only on the tume interval $t=s_{2}-s_{1}$. Substitution of eq. (4) into eq. (11) gives

$$
D_{\mathrm{s}}=\frac{1}{6}\left\langle\int_{-t}^{t} \mathrm{~d} \tau\left[v_{1}(0) \cdot v_{1}(\tau)+v_{1}(0) \cdot v_{\mathrm{B}}(\tau)+v_{\mathrm{B}}(0) \cdot v_{1}(\tau)+v_{\mathrm{B}}(0) \cdot v_{\mathrm{B}}(\tau)\right](1-|\tau| / t)\right\rangle .
$$

Here the low-frequency $(t \rightarrow \infty)$ lımit is considered. Substitution of eqs. (4), (8) and (9) into (12) leads to

$$
\begin{aligned}
D_{\mathrm{s}}= & \frac{1}{6}\left\langle\int_{-\infty}^{\infty} \mathrm{d} \tau v_{1}(0) \cdot v_{1}(\tau)+\int_{-\infty}^{0} \mathrm{~d} \tau v_{1}(0) \cdot v_{\mathrm{B}}(\tau)-\int_{0}^{\infty} \mathrm{d} \tau v_{\mathrm{B}}(0) \cdot v_{\mathrm{B}}(\tau)\right. \\
& \left.+\int_{-\infty}^{\infty} \mathrm{d} \tau\left[-v_{1}(0) \cdot v_{1}(\tau)+\delta v_{\mathrm{B}}(0) \cdot v_{1}(\tau)+v_{\mathrm{B}}(0) \cdot v_{\mathrm{B}}(\tau)\right]\right\rangle
\end{aligned}
$$

In eq. (13), the first and fourth terms of the right-hand-side cancel. The third term has a correlation time $\tau_{\mathrm{B}}$; since $\bar{v}_{\mathrm{B}}$ is typically much less than $v_{\mathrm{B}}$, the third term is negligible by comparison with the sixth. In interpreting the fifth term, the induced flow of the solute does not result in stress fluctuations in the solvent, so this term must vanish for $\tau<0$. The inverse does occur; brownian motion can move particles into configurations in which $v_{1} \neq 0$. The fifth term therefore does not vanish for $\tau>0$, time translation 
invariance permits a merger of the second and fifth terms. Invoking the defunition (2) of $f$, in terms of $D_{、}$, one finds

$$
D_{1} \equiv \frac{k_{\mathrm{B}} T}{f_{v}}=\frac{1}{6}\left\langle\int_{-\infty}^{\infty} v_{\mathrm{B}}(0) \cdot v_{\mathrm{B}}(t) \mathrm{d} t+2 \int_{-\infty}^{0} \mathrm{~d} t v_{\mathrm{B}}(t) \cdot v_{\mathrm{l}}(0)\right\rangle
$$

From the usual theory of brownian motion for particles without hydrodynamic interactions.

$$
6 D_{0}=\left\langle\int_{-\infty}^{\infty} v_{\mathrm{B}}(0) \cdot v_{\mathrm{B}}(t) \mathrm{d} t\right\rangle
$$

so

$$
D_{\mathrm{s}}=D_{0}\left[1+\frac{2}{D_{0}} \int_{-\infty}^{0} \mathrm{~d} t\left\langle v_{\mathrm{B}}(t) \cdot v_{\mathrm{l}}(0)\right\rangle\right] .
$$

The final integral in eq (16) represents the modification of the self-diffusion coefficient by direct interactions. This integral is identical to the one previously obtained in ref. [7] for the modification of the friction factor of the mutual-diffusion coefficient by direct interactions. For the specific case of particles with a weak gaussian interaction such that their radial distribution function $g(r)$ is

$$
g(r)=1+B_{0} \exp \left(-r^{2} / r_{0}^{2}\right) / 8 \pi^{3 / 2},
$$

it was found that

$$
\left\langle 2 \int_{-\infty}^{0} \mathrm{~d} t v_{\mathrm{B}}(t) \cdot v_{1}(0)\right\rangle=-0.3917 D_{0} \dot{\phi}_{T}|g(0)-1| / 8 \sqrt{2}
$$

where $\phi=c V, c$ is the macroparticle number concentration. $V=4\left|B_{0}\right| r_{0}^{3}$ is the effective volume of a particle. $B_{0}$ is an interaction strength parameter, and $r_{0}$ is an effective range for the interaction. [The dispute between ref. [7] and refs. [4-6] over the modification of $f_{\mathrm{m}}$ by direct interactions could be said to involve a disagreement as to whether that modification is given by the final integral in eq (16) or by the similar form

$$
\sum_{t, j=1}^{N} \int_{-\infty}^{0} \mathrm{~d} t v_{\mathrm{B} t}(t) v_{1,}(0)
$$

the double sum being over all $N$ particles in the system. This double sum mas be argued to vanish identically. If (19) were the correct form. it would appear that direct interactions would not modify $f_{\mathrm{m}}$. It is not presently disputed that eqs. (16) and (17) lead to eq. (18).]

\section{Discussion}

In the above, a Langevin-type approach has been used to calculate the contribution of direct interactions to the self-diffusion coefficient $D_{s}$. It was found that direct interactions lead to a concentration dependence of $D_{s}$ even if there are no hydrodynamic forces present. This result is in qualitative agreement with the results of Mazo [9] and Marqusee and Deutch [16]. who found from fluctuation-dissipation and Mori theory arguments that direct interactions lead to a concentration dependence of $D$. even if hydrodynamic forces are not considered. Our results differ from those presented previously in that this paper used a Langevin-type approach, similar to that used by Pusey and Tough [13]. rather than the generalized Smoluchowski equation

$$
\partial P\left(\boldsymbol{r}^{N}, t\right) / \partial t=\sum_{t}\left[D_{0} \nabla_{t}^{2}-\nabla_{l} F_{l} / f\right] P\left(\boldsymbol{r}^{\wedge}, t\right)
$$


A qualitative image of the mechanism whereby direct interactıons reduce the self-diffusion coefficient may be of some interest. In the absence of direct interactions, the Langevin model indicates that each particle is acted on by a drag force $-f v$ and a fuctuating force $F_{1}(t)$. If one adds direct interactions between neigboring brownian macroparticles. a brownian particle $i$ which attempts to move a substantial distance suffers encounters with its neighbors Through these encounters, the fluctuating force $F$, on the moving particle is effectively dispersed over some number $n$ of neighbors, so that the particle motion is opposed by a drag coefficient $\approx n f$, reducing the displacement of the particle form the displacement which it would have experienced if direct interactions had not been present. This resistance to the motion of a particle is rather like the "cage model" of Pusey [15]. By symmetry. particle $t$, while dispersing the force $F$, of itself over $n$ neighbors, will also on the average receive a fraction $1 / n$ of the random forces $F_{1} \cdots F_{n}$ on each of its neighbors. Since the random forces $F_{1} \cdots F_{n}, F_{1}$ are uncorrelated. the total force on particle t grows only as $n^{1 / 2}$, in contrast to the total drag coefficient. which is proportional to $n^{1}$. The self-diffusion coefficient $D_{s}$ depends on the mean-square ratio of the force to the drag coefficient. so one qualitatively predicts $D \approx n^{-1}$. For the special case in which the "interparticle" interaction is a covalent bond. this prediction is exact. In the absence of hydrodynaml interactions. the diffusion coefficient of an n-mer is expected to be precisely $1 / n$ of the diffusion coefficient of the corresponding noonomer.

An alternative explanation for part of the fallure of refs. [4-6] to identify any contribution of direct interactions to $f_{\mathrm{n}}$ may lie in the calculational basis used in some of that work. Refs [4-6] are in part based on the generalized Smoluchowski equation (20). The generalized Smoluchowski equation was originally used to calculate the diffusion of macroparticles in the presence of rigid walls and constant external potentials, as encountered. e $g$ in the sedimentation problem [17]. In these onginal papers. the movement of a given brownian particle has no effect on the position of walls or the spatial dependence of the external potential, so the walls and potentials were fixed and determinate. In a solution of interacting brownian particles, the motion of each particle can affect the motions of the other particles. so that the potential encountered by a diffusing particle is not a determinate function of that particle's path in space. The consequences of this difference may be seen by calculating the initial slope of the dynamic structure factor $S(k, t)$ measured by light-scattering spectroscopy, either by using the generalized Smoluchoswki equation

$$
\left.\frac{\mathrm{d} S(k, t)}{\mathrm{d} t}\right|_{t \rightarrow 0}=\left\langle\frac{1}{N} \sum_{t, j=1}^{N} \exp \left\{\mathrm{i} k \cdot\left[r_{t}(0)-r_{j}(0)\right]\right\}\left(-D_{0} k^{2}-\mathrm{i} k \cdot \nabla_{t} W^{2} / f\right)\right\rangle .
$$

or by using the Langevin equation

$$
\begin{aligned}
\left.\frac{\mathrm{d} S(k, t)}{\mathrm{d} t}\right|_{t \rightarrow 0}= & \left\langle\frac{1}{N} \sum_{t=1}^{N} \exp \left\{\mathrm{i} k \cdot\left[r_{t}(0)-r_{j}(0)\right]\right\}\right. \\
& \left.\times\left[-k^{2}: \int_{0}^{t}\left[v_{\mathrm{B} t}(s) v_{\mathrm{B} t}(t)+v_{\mathrm{B} t}(s) v_{1}(t)\right] \mathrm{d} s+\mathrm{i} k \cdot v_{t 1}(t)\right]\right\rangle .
\end{aligned}
$$

Eq. (22) is the same as eq. (20) or ref. [7]. These equations would agree with each other if one had

$$
D_{0} k^{2}=\int_{0}^{t} \mathrm{~d} s\left[k \cdot v_{\mathrm{B}}(s) k \cdot v_{\mathrm{B}}(t)+k \cdot v_{\mathrm{B} t}(s) k \cdot v_{\mathrm{L}}(t)\right] \text {. }
$$

However, in eq (21) $D_{0}$ has consistently been interpreted as the bare diffusion coefficient of eq. (15), while from (23) one concludes that the $D_{0}$ of the generalized Smoluchowski equation is properly a "dressed" diffusion coefficient, in which the direct interactions have perturbed the diffusion rate. Calculatıons based on the generalized Smoluchowski equation and the identification of $D_{0}$ as the free-particle diffusion coefficient should therefore predict that the effect treated here does not contribute to $f_{m}$. 


\section{References}

[1] G.D.J Phillies J Chem Phys 60 (1974) 976

[2] R S Hall and C S Johnson Jr. J Chem Phys 72 (1980) 4251

[3] R S Hall. Y S Oh and C S Johnson. Jr. J Phys Chem 84 (1980) 756

[4] B J Acherson J Chem Phys 69 (1978) 684

[5] W Dieterich and I Peschel. Physica A95 (1979) 208

[6] I A Marqusee. Ph D Thesis MIT (1981)

[7] G D J. Philiıes J Chem Phys 74 (1981) 2436

[S] J G Kirkuood and J. Riseman J Chem Phys 16 (1948) 565

[9] R M Mazo. J Chem Phys 43 (1965) 2873 J Statist Phys 1 (1969) 59.

[10] V.I. Deuln Mahromol Chem 180 (1979) 263.

[11] G.D.J. Phillies. J. Chem Phıs 67 (1977) 4690.71 (1979) 1492.

[12] S A Rice and P Gray. The statistical mechanics of simple higuts (W/le-Interscience Vieu Yorh. 1972) ch 4

[13] PN Pusey and R J A Tough J Phys A 15 (1982) 1291

[14] G K. Batchelor. J. Fluid Mech 74 (1976) 1

[15] P N. Pusey. J. Phys A11 (1981) 119

[16] I A Marqusee and $J$ is Deutch J. Chem Phys 73 (1980) 5396

[17] N Wax Selected papers on noise and stochastic processes (Dover Neu York 1954) especially the paperv by $S$ Chandraskhar and by MC Wang and $G E$. Uhlenbech 